\title{
análissis automático en la industria del cemento por espectrometría de rayos $X$ y calculador asociado
}

M. VON EUW

Director en la Sociedad de Ciments Français

Revue des Matériaux, n.` 624, septiembre 1967

La Sociedad de CIMENTS FRANÇAIS emplea desde hace tres años, en su Laboratorio Central de Levallois, un espectrómetro automático de rayos $X, P W$ 1210. Las materias controladas son los productos empleados y fabricados corrientemente en la industria del cemento.

Se han hecho patrones por fusión de mezclas puras. Estos patrones han permitido calcular para cada elemento la ecuación de una recta de referencia, a partir de la cual se efectúan los cálculos que dan la composición elemental de las muestras analizadas.

Estos cálculos necesitan dos operadores, y los procesos verbales de análisis deben ser dactilografiados enseguida.

Se ha considerado útil asociar al espectrómetro un calculador $P R$ 8000, que realiza el conjunto de los cálculos y la redacción de los procesos verbales de análisis.

Con este conjunto, se llega a un trabajo de análisis completamente automatizado entre la introducción de la muestra en el espectrómetro y la salida de los resultados imprimidos. Gracias al calculador a él asociado, el espectrómetro puede ser utilizado a pleno rendimiento.

\section{introducción}

La industria cementera hace mucho uso de los análisis químicos en cada fase de la elaboración de sus productos, incluido el con^rol en las obras donde se ha de emplear:

- investigaciones sobre nuevas canteras;

- estudio de canteras en explotación;

- preparación de mezclas antes de la cocción;

- control de los clínkeres a la salida de los hornos; 
- control de los cementos a la salida de los molinos y en su expedición;

- control de los cementos vendidos en los almacenes o en las obras.

Las materias primas son, principalmente, arcillas, margas y calizas; se les añade, a veces, pequeñas cantidades de bauxita, pirita tostada o sílice.

Por los análisis debe conocerse el contenido de los siguientes elementos, expresados en óxidos según la práctica cementera:

$\mathrm{SiO}_{2}-\mathrm{Al}_{2} \mathrm{O}_{3}-\mathrm{Fe}_{2} \mathrm{O}_{3}-\mathrm{TiO}_{2}-\mathrm{MnO}-\mathrm{CaO}-\mathrm{MgO}-\mathrm{SO}_{3}-\mathrm{K}_{2} \mathrm{O}-\mathrm{P}_{2} \mathrm{O}_{5}$.

La composición química de las mezclas preparadas antes de la cocción se elije después de haber sido convenientemente estudiada; juega en efecto un papel esencial:

- en primer lugar, en el reglaje y funcionamiento regular de los hornos;

- más tarde, en la calidad de los cementos fabricados.

$\mathrm{El}$ buen funcionamiento de los hornos depende de las proporciones relativas de $\mathrm{SiO}_{2}$, $\mathrm{Al}_{2} \mathrm{O}_{3}, \mathrm{Fe}_{2} \mathrm{O}_{3}$ y $\mathrm{CaO}$ y de los contenidos en $\mathrm{SO}_{3}$ y $\mathrm{K}_{2} \mathrm{O}$; de hecho la temperatura de clinkerización viene fijada por la composición de las mezclas, y la formación de costras y anillos que obstruyen accidentalmente la sección de los hornos resulta muchas veces de la presencia de sulfatos alcalinos en proporciones muy importantes.

Durante la cocción los constituyentes de las mezclas crudas sufren una serie de transformaciones que conducen, en la zona de clinkerización, a la formación de silicatos y aluminatos de calcio. Estos silicatos y aluminatos son los compuestos activos de los cementos; por reacción química con el agua dan sales hidratadas cuyos microcristales se sueldan los unos a los otros, así como a los áridos, confiriendo a los morteros y hormigones sus características físicas y mecánicas.

Estas generalidades justifican el papel del laboratorio de análisis en una fábrica de cemento: el control de muestras debe ser numeroso y efectuarse con rapidez, lo que necesita métodos y aparatos bien adaptados.

Hace 10 ó 15 años un análisis químico completo no podía obtenerse hasta 2 días de manipulaciones más o menos delicadas. Desde entonces los métodos químicos se han perfeccionado y es posible hacer un análisis en unas horas; sin embargo, en muchos casos, este plazo es todavía muy largo, pues la concepción de las modernas fábricas y la importancia de los tonelajes fabricados conducen a un control intensivo que va desde la toma de muestras cada $1 / 2$ hora o cada $1 / 4$ de hora, por ejemplo, al control continuo.

No siendo los métodos químicos lo bastante rápidos como para obtener análisis con esta cadencia, los laboratorios recurren cada vez más a los métodos físicos, entre los cuales la fluorescencia de rayos $\mathrm{X}$ tiene uno de los lugares más importantes.

Para comprender mejor lo que se pide al laboratorio de una fábrica de cemento, es preciso distinguir dos casos:

- el laboratorio de fábrica, que regula la fabricación;

- y el laboratorio central de control.

En el caso de controlar la fabricación, no se analiza generalmente más que una sola muestra, aunque es preciso conocer los resultados lo más deprisa posible; es lo que se hace en la preparación de mezclas crudas por vía seca, ajustándose las proporciones de ca- 
liza y arcilla, en todo momento, a la entrada del molino. Con este proceder el silo de materia preparada, cuya capacidad puede alcanzar 2.000 t, debe tener la composición prevista sin realizar correcciones.

Las fábricas que han adoptado la espectrometría de rayos $\mathrm{X}$ para realizar este trabajo emplean con preferencia aparatos con varios canales que permiten la dosificación simultánea de algunos elementos esenciales, tales como: $\mathrm{Si}-\mathrm{Al}-\mathrm{Fe}-\mathrm{Ca}$.

El laboratorio central de control tiene un objetivo completamente distinto: verifica generalmente la composición de un gran número de muestras de orígenes muy diverso, que van desde materias primas a los diferentes tipos de cemento.

En la Sociedad de Ciments Français, el laboratorio central controla diariamente los productos procedentes de 14 fábricas, lo que representa un programa de 60 a 80 análisis por día; esto se consigue con ayuda de un espectrómetro automático PW 1210 al que se ha asociado un calculador numérico PR 8000.

\section{método de análisis}

El método de análisis se publicó ya, en colaboración con M. Merx, con ocasión de las jornadas de estudios del C.E.R.I.L.H. en enero de 1964; de él se recordarán los puntos esenciales:

1). A fin de eliminar los efectos de estructura, que se sufren muy a menudo trabajando sobre polvos compactados, las muestras se vitrifican por fusión con tetraborato de litio $\left(\mathrm{Li}_{2} \mathrm{~B}_{4} \mathrm{O}_{7}\right)$.

2). Para atenuar los efectos interelementos, la vitrificación se hace con adición de un elemento peșado; se utiliza el óxido de lantano $\left(\mathrm{La}_{2} \mathrm{O}_{3}\right)$.

La composición de las mezclas fundidas a $1.200^{\circ} \mathrm{C}$ en crisol de platino es la siguiente:

1 parte de muestra;

1 parte de óxido de lantano;

4 partes de tetraborato de litio.

La fusión dura 10 minutos; generalmente se opera sobre series de 6 ó 12 muestras, en un horno eléctrico regulado.

En principio, un operador se encarga de las pesadas y un segundo operador hace las perlas y limpia los crisoles.

3). La dosificación de los diversos elementos se hace con referencia a patrones sintéticos preparados con ayuda de productos químicamente puros.

A este propósito, el autor quiere recordar a los que siguen la evolución de la espectrometría de rayos $\mathrm{X}$ desde su principio, que en el coloquio de Grenoble en 1959, se habían imputado los desacuerdos entre el análisis químico y el análisis por rayos $\mathrm{X}$, a los métodos químicos; M. Hans, en particular, lo había subrayado a propósito de los controles en metalurgia.

El autor de este trabajo se dio cuenta de que el único método de valorar la precisión de la espectrometría de rayos X era suprimir la referencia a los resultados de los mé- 
todos químicos y proceder a un calibrado a partir de productos químicamente puros. Las dispersiones estadísticas de los métodos químicos son muy grandes, como lo evidencian los resultados de los ensayos mancomunados organizados cada año por el C.E.R.I.L.H., en los que participan todos los iaboratorios franceses que se ocupan de cemento; de los resultados del año 1966, se han retenido los siguientes valores:

\begin{tabular}{|l|c|c|c|c|c|}
\hline & $\mathrm{SiO}_{2}$ & $\mathrm{Al}_{2} \mathrm{O}_{3}$ & $\mathrm{Fe}_{2} \mathrm{O}_{3}$ & $\mathrm{CaO}$ & $\mathrm{MgO}$ \\
\hline Núm. de resultados & 107 & 49 & 111 & 107 & 105 \\
\hline Valor mínimo & 21,43 & 6,85 & 1,50 & 54,43 & 0,34 \\
\hline Valor máximo & 23,77 & 7,90 & 3,00 & 62,09 & 2,59 \\
\hline Amplitud & 2,34 & 1,05 & 1,50 & 7,66 & 2,25 \\
\hline Desviación stand. & 0,39 & 0,17 & 0,24 & 0,72 & 0,30 \\
\hline
\end{tabular}

Estos ejemplos demuestran claramente que las críticás hechas al análisis por rayos $\mathrm{X}$ en los primeros ensayos sobre los cementos proceden en gran parte de un optimismo exagerado de los químicos en cuanto a sus propios métodos de análisis.

El autor considera que en el interior de un mismo laboratorio, los métodos químicos correctamente aplicados dan resultados mejor agrupados que los de los ensayos comunes del C.E.R.I.L.H.; esto se debe probablemente a que ciertos participantes en estos ensayos no practican corrientemente los análisis considerados.

La tabla núm. 2 da las ecuaciones de las rectas de referencia establecidas con ayuda de 40 mezclas de productos químicamente puros. Los $2 / 3$ de estas mezclas cubren la zona de composición de los cementos y $1 / 3$ de ellas se refiere a la zona de composición de las margas y arcillas. Puede observarse que los efectos interelementos se han eliminado prácticamente con el óxido de lantano, salvo para la dosificación del hierro en las margas: en este caso se ha aportado una pequeña corrección para tener en cuenta el calcio.

Es de señalar que en los diez elementos controlados, se ha renunciado a dosificar $\mathrm{Mg}$ y $\mathrm{S}$, empleando las perlas vitrificadas:

- los contenidos generalmente pequeños de $\mathrm{Mg}$ y la dilución con los fundentes reducen la precisión de la dosificación de este elemento, el más ligero que entra en el análisis;

- en lo que concierne al $\mathrm{S}$, hay una pequeña volatilización durante la fusión de la perla.

Por lo tanto se opera por separado con polvo comprimido para estos dos elementos.

\section{empleo del espectrómetro automático}

El espectrómetro PW 1210 empleado en el Laboratorio Central de Ciments Français está equipado de un tubo con ánodo de cromo de $2 \mathrm{~kW}$, funcionando con $60 \mathrm{~kW}$ y $24 \mathrm{~mA}$. 
La preselección de las condiciones operatorias para el análisis automático se efectúa sobre un panel programa que lleva consigo 15 posibles posiciones para el goniómetro ; cada posición angular corresponde al ángulo característico elegido para un elemento a dosificar.

La tabla núm. 1 resume las condiciones de reglaje predeterminadas para cada elemento; una vez anunciadas estas condiciones, el modo de operar es el siguiente:

1. El método de medida adoptado es el del informe; consiste en emplear una muestra-testigo para corregir los errores instrumentales.

El aparato fija el tiempo necesario para recoger un número predeterminado de impulsos del testigo para un elemento dado; cada muestra será irradiada este mismo tiempo. Se expresa entonces la relación entre el número de impulsos detectados y el número de impulsos establecidos para el testigo.

El operador coloca las muestras en los cuatro alojamientos del soporte que gira en el interior de un recipiente con vacío. El primero de estos alojamientos está ocupado por la muestra-testigo que se mantiene en su sitio durante todos los análisis.

2. Estando cerrada la tapa del recipiente, el operador pulsa el botón de ARRANQUE; a partir de este momento todas las operaciones se hacen automáticamente de acuerdo con el programa anunciado.

3. El vacío se realiza en el recipiente con una bomba de paletas.

4. Los contadores fijados sobre el sistema goniométrico se colocan en la primera posición angular elegida. La radiación característica se selecciona por el cristal analizador antes de alcanzar el contador de flujo gaseoso empleado para todos los elementos que nos interesan. Hay que señalar que para la dosificación del manganeso el recuento se hace simultáneamente por el contador de flujo y el centelleador colocados en serie.

5. El contador permanece sobre la primera posición angular mientras que cada una de las muestras se coloca en posición de irradiación.

Una vez terminados los recuentos para el primer elemento y en las cuatro muestras, el contador se coloca en la posición angular siguiente, continuando así el ciclo hasta el último elemento.

6. El espectrómetro lleva consigo circuitos de recuento y de integraciọn que efectúan la medida del número de impulsos emitidos por el contador y la transmiten a un aparato de registro.

7. Los tiempos de recuento de los diversos elementos varía de 12 segundos para el titanio a dos veces 80 segundos para el magnesio, siendo doblado el tiempo exigido para este último elemento por la medida del fondo continuo.

Reuniendo todas las operaciones, el tiempo global de un ciclo es de 17 minutos para la determinación de los siguientes elementos: $\mathrm{Si}, \mathrm{Al}, \mathrm{Fe}, \mathrm{Ti}, \mathrm{Mn}, \mathrm{Ca}, \mathrm{K}, \mathrm{P}$. Este tiempo se aplica al paso del testigo y de las tres muestras colocadas en el espectrómetro; comprende la colocación, la demora del vacío, la rotación del portamuestras y la del goniómetro.

A esta duración del análisis sobre perla vitrificada, es preciso añadir los tiempos de determinación del azufre y del magnesio efectuados separadamente sobre polvo compactado en 12 minutos para las tres muestras, lo que conduce a un tiempo para los tres análisis de 29 minutos, es decir, unos 10 minutos por muestra. 


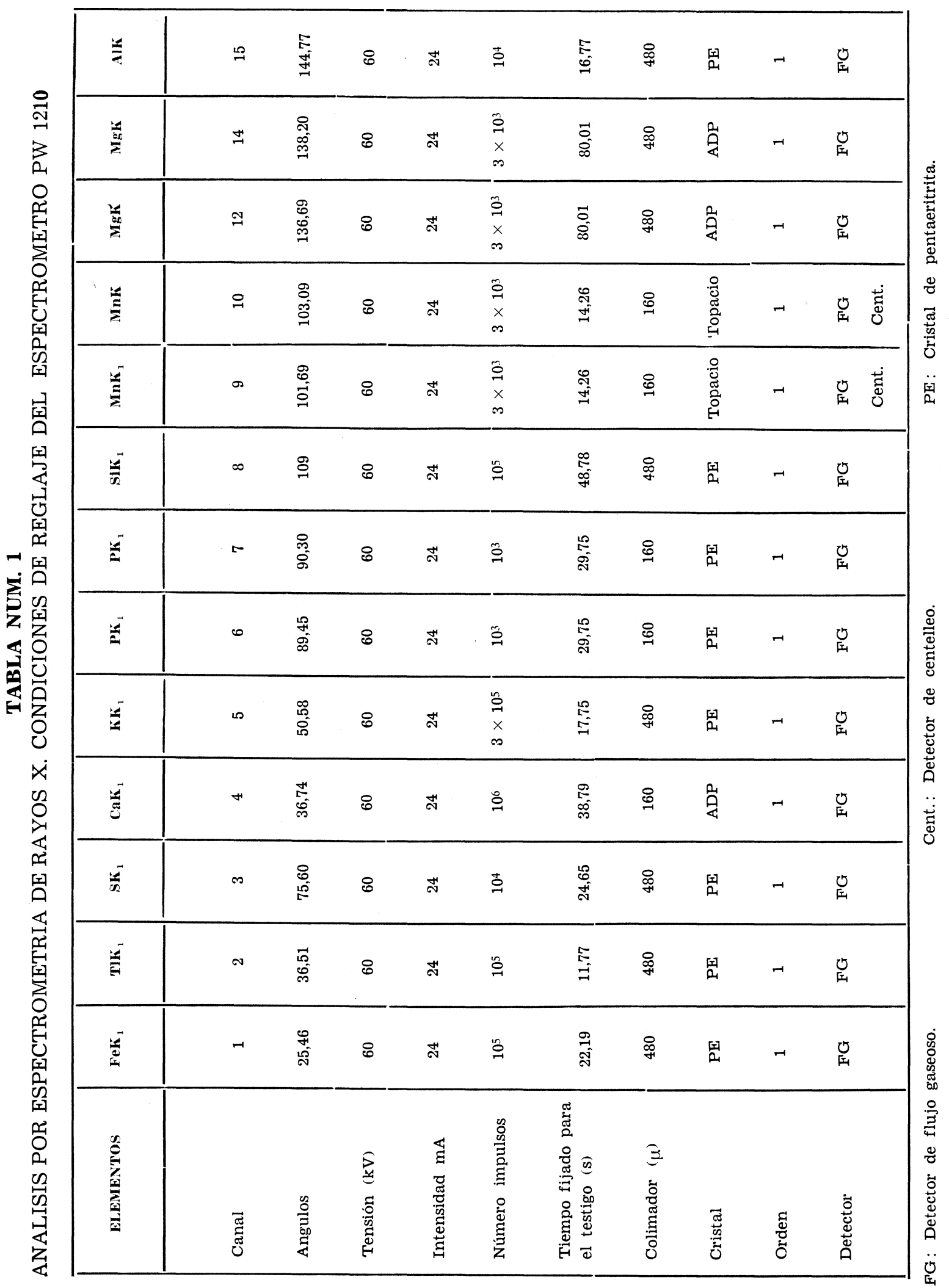


TABLA NUM. 2

ANALISIS POR ESPECTROMETRIA DE RAYOS X

Ecuaciones de las rectas de referencia

\begin{tabular}{|c|c|c|c|}
\hline Canales & Elementos & Margas $\mathrm{y}$ arcillas & Cementos \\
\hline 1 & $\mathrm{Fe}_{2} \mathrm{O}_{3}$ & $\frac{\mathrm{I}_{\mathrm{Fe}}-22.307+252 \mathrm{CaO}}{18.312}$ & $\frac{\mathrm{I}_{\mathrm{Fe}}-5.657}{18.312}$ \\
\hline 2 & $\mathrm{TiO}_{2}$ & $\frac{\mathrm{I}_{\mathrm{Ti}}-36.569}{200.773}$ & $\frac{I_{\mathrm{Ti}}-36.569}{200.773}$ \\
\hline 3 & $\mathrm{SO}_{3}$ & $\frac{I_{s}+8.477}{46.907}$ & $\frac{I_{S}+8.477}{46.907}$ \\
\hline 4 & $\mathrm{CaO}$ & $\frac{\mathrm{I}_{\mathrm{Ca}}-4.289}{16.008}$ & $\frac{I_{\mathrm{Ca}}-46.205}{14.952}$ \\
\hline 5 & $\mathrm{~K} O$ & $\frac{I_{K}-4.388}{89.083}$ & $\frac{I_{K}-4.388}{89.083}$ \\
\hline $6-7$ & $\mathbf{P}_{2} \mathbf{O}_{5}$ & $\frac{I_{P}-6}{500}$ & $\frac{I_{P}-6}{500}$ \\
\hline 8 & $\mathrm{SiO}_{2}$ & $\frac{\mathrm{I}_{\mathrm{Si}}-1.804}{2.900}$ & $\frac{\mathrm{I}_{\mathrm{Si}}-1.804}{2.900}$ \\
\hline $9-10$ & MnO & $\frac{I_{M n}+65}{512}$ & $\frac{I_{\mathrm{Mn}}+65}{512}$ \\
\hline $12-14$ & $\mathrm{MgO}$ & $\frac{I_{M g}+588}{1.472}$ & $\frac{\mathrm{I}_{\mathrm{Mg}}+588}{1.472}$ \\
\hline 15 & $\mathrm{Al}_{2} \mathrm{O}_{3}$ & $\frac{I_{A 1}+4.270}{712}$ & $\frac{I_{\mathrm{A} 1}-386}{457}$ \\
\hline
\end{tabular}

8. Desde hace 3 años que el espectrómetro está en servicio, y hasta principios de este año, el operador leía los recuentos y aplicaba las fórmulas de la tabla núm. 1 para calcular los análisis brutos de las perlas, es decir, de las muestras consideradas sin pérdida al fuego.

Una segunda persona recogía estos análisis brutos y los manejaba considerando determinaciones químicas realizadas por otros, tales como las pérdidas al fuego o los contenidos en silice insoluble. Con ayuda de una máquina de calcular se obtenían los diversos coeficientes empleados en las fábricas de cemento:

- saturación en cal;

- módulos silícico y alumino-férrico;

- módulo hidráulico;

- composición potencial en diferentes condiciones de enfriamiento de los clínkeres. 
Una tercera persona se ocupaba de dactilografiar los boletines de análisis antes de transmitirlos al ingeniero encargado del control y más tarde a las fábricas interesadas con las observaciones correspondientes.

\section{empleo del calculador PR 8000}

El manejo de los recuentos dados por el espectrómetro representaba hasta principios de este año un trabajo considerable. El personal era incapaz de seguir el ritmo de la máquina de la que no se utilizaba más que en parte todas sus posibilidades: en tanto que la capacidad de tratamiento del PW 1210 es de 9 análisis de cemento o productos con él relacionados por hora, el laboratorio sólo podía producir por término medio unos 30 análisis por día.

La adquisición del calculador electrónico debía mejorar considerablemente el ritmo del control, aprovechando al máximo las posibilidades del espectrómetro.

El PR 8000 en servicio desde enero último se compone:

1. De una unidad central cuya memoria, constituida por toros de ferrita de coeficiente de temperatura muy bajo, tienen una capacidad de 4.096 palabras, teniendo cada palabra una longitud de 24 posiciones binarias.

2. De una unidad de adaptación al espectrómetro. Esta unidad emite y recibe las señales de sincronización permitiendo la adquisición de los recuentos emitidos por el espectrómetro.

3. De una máquina de escribir que imprime automáticamente los resultados $d \epsilon$ cálculo con una cadencia de impresión de 15 caracteres por segundo. Permite al operador "dialogar" con el calculador, introduciendo manualmente ciertos datos o dirigiendo la ejecución del programa elegido.

4. De un lector de banda perforada cuya función es permitir la introducción del programa de tratamiento de medidas en la memoria del calculador. Su velocidad de lectura es de 120 caracteres/segundo.

5. De un perforador de bandas que trabaja a 60 caracteres/segundo. Sirve de almacenador de resultados de cálculo con vistas a un tratamiento posterior para realizar estudios estadísticos.

El primer trabajo que ha habido que hacer antes de poner el calculador en servicio ha sido el establecimiento de un programa. Este programa debía permitir la impresión de boletines de análisis completamente calculados y correctamente puestos en su página para todos los materiales controlados por el laboratorio.

Con el fin de aplicar en cada caso los cálculos correspondientes, se ha establecido primero un organigrama, reproducido en la tabla núm. 3. Este organigrama indica el encadenamiento de los tratamientos que debe efectuar el calculador:

- adquisición de la designación de la muestra y de los datos químicos. Esta adquisición se hace manualmente por el operador con ayuda de la máquina de escribir ;

- adquisición de los datos procedentes del espectrómetro por medio de la unidad de adaptación; 
TABLA NUM. 3

ORGANIZACION GENERAL DEL PROGRAMA DE ANALISIS

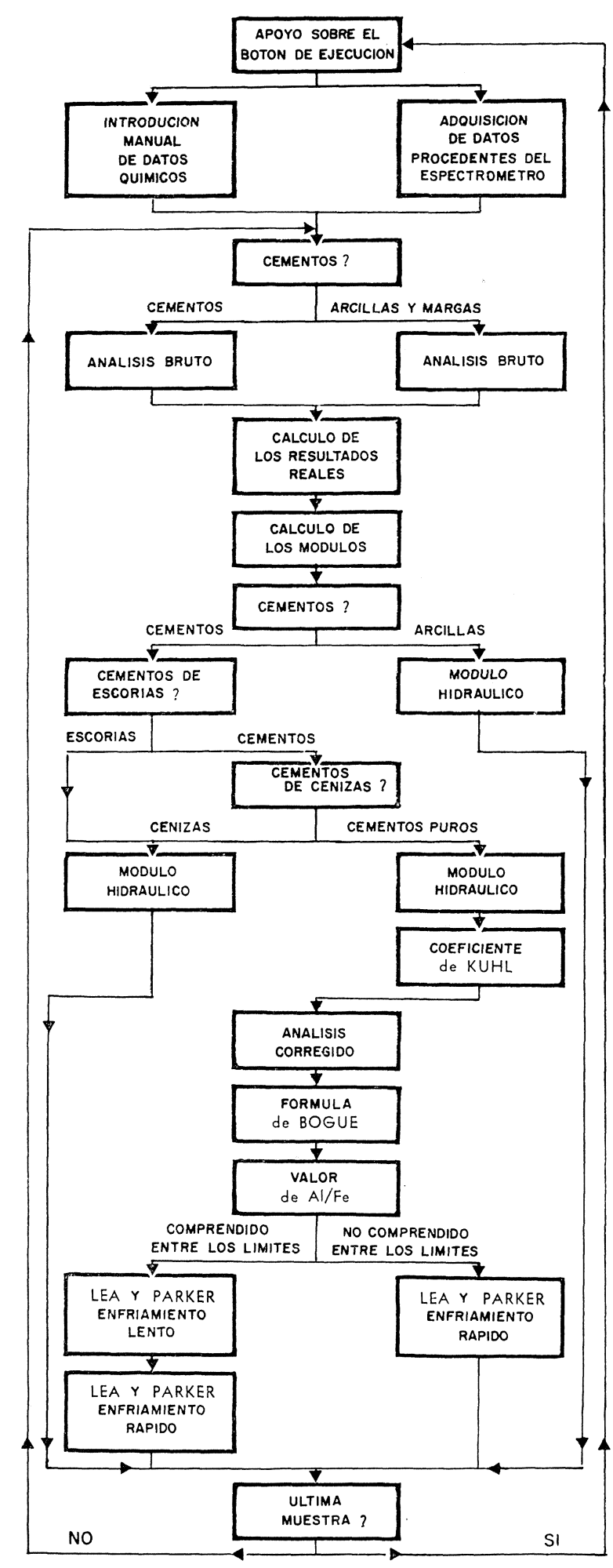

- clasificación según la naturaleza de las muestras para la elección de las fórmulas de calibrado a emplear en el cálculo de los contenidos;

- cálculo del análisis bruto de la perla ;

- cálculo del análisis referido a la muestra real;

- elección de los coeficientes de cálculo, según el origen de las muestras;

- cálculo de las composiciones potenciales;

- número de duplicados de los boletines de análisis.

Habiéndose establecido desde un principio este organigrama, se ha redactado un programa en lenguaje máquina. El programa, a su vez, se ha trascrito en banda perforada. La introducción de esta banda en el calculador por el lector exige $3 \mathrm{minu}$ tos; permanece en la memoria y no es necesario reintroducirla, salvo si una falsa maniobra llega a borrar las memorias.

Una vez en su lugar el programa de tratamiento, el calculador recibe las informaciones manuales y las procedentes automáticamente del espectrómetro. Cuando el ciclo de análisis está terminado, el calculador entrega una factura dacîilografiada en la cual figuran todos los informes que definen la muestra y los resultados de los cálculos. El número de duplicados ha sido fijado por el programa.

La tabla núm. 4 es un ejemplo del boletín de análisis: el tiempo de impresión de este boletín por la máquina es de unos 30 segundos. Es necesario señalar que se puede volver a hacer un nuevo ciclo de análisis con otras muestras sin esperar a que estén acabados de imprimir los boletines. 
TABLA NUM. 4

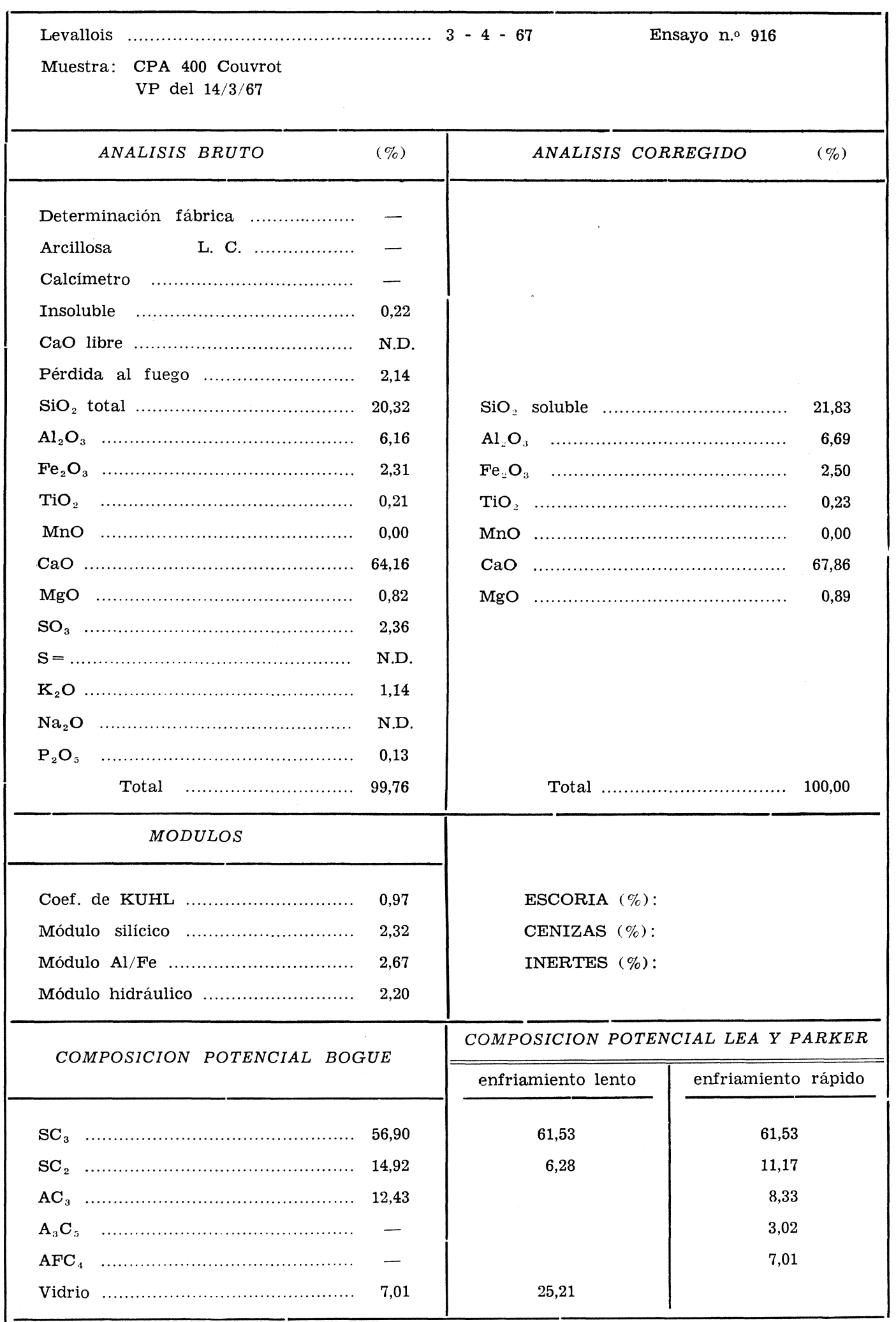


Así, gracias al calculador, el espectrómetro puede emplearse a pleno rendimiento y expedir de 6 a 9 análisis por hora, según el número de elementos determinados, cálculos efectuados y boletines dactilografiados.

\section{precisión de los resultados}

A fin de cifrar la dispersión de los resultados se ha procedido a un estudio estadístico analizando 20 veces la misma muestra con ayuda de 20 perlas diferentes y pasando igualmente 20 veces una misma perla a razón de una vez por día.

Los resultados para las 20 perlas de la misma muestra de cemento son los siguientes:

\begin{tabular}{|c|c|c|c|c|c|}
\hline & $\begin{array}{l}\text { Media } \\
\bar{x}\end{array}$ & $\begin{array}{c}\text { Extensión } \\
\qquad \mathrm{E}\end{array}$ & $\begin{array}{c}\text { Desviación } \\
\text { standard } \\
\sigma\end{array}$ & $\begin{array}{c}\text { Coeficiente } \\
\text { de } \\
\text { variación }\end{array}$ & $\begin{array}{c}\text { Dispersión } \\
2 \sigma\end{array}$ \\
\hline $\mathrm{SiO}_{2}$ & 20,66 & 0,32 & 0,10 & 0,5 & $\pm 0,20$ \\
\hline $\mathrm{Al}_{2} \mathrm{O}_{3}$ & 4,60 & 0,28 & 0,08 & 1,7 & 0,16 \\
\hline $\mathrm{Fe}_{2} \mathrm{O}_{3}$ & 3,06 & 0,07 & 0,02 & 0,6 & 0,04 \\
\hline $\mathrm{TiO}_{2}$ & 0,19 & 0,01 & 0 & & \\
\hline $\mathrm{MnO}$ & 0,10 & 0,15 & 0,04 & 38,8 & 0,08 \\
\hline $\mathrm{CaO}$ & 62,89 & 0,65 & 0,22 & 0,3 & 0,44 \\
\hline $\mathrm{MgO}$ & 0,97 & 0,43 & 0,12 & 12,6 & 0,24 \\
\hline $\mathrm{SO}_{3}$ & 2,56 & 0,27 & 0,09 & 3,4 & 0,18 \\
\hline $\mathrm{K}_{2} \mathrm{O}$ & 1,48 & 0,12 & 0,04 & 2,7 & 0,08 \\
\hline $\mathrm{P}_{2} \mathrm{O}_{5}$ & 0,32 & 0,21 & 0,06 & 18,7 & 0,12 \\
\hline
\end{tabular}

La dispersión indicada para cada elemento es igual a $2 \sigma$, lo que significa que el $95 \%$ de los resultados están comprendidos entre valores que no difieren $2 \sigma$ en más o en menos del valor encontrado.

La estadística hecha sobre 20 veces la misma perla de una dispersión idéntica para todos los elementos, salvo para la cal, en que la precisión de la dosificación se mejora con $2 \sigma=0,24$ en lugar de 0,44. Esta nota tiende a demostrar que el estado de la perla, que puede variar de una preparación a otra, condiciona la dispersión de los resultados de ciertas determinaciones. 


\section{conclusión}

Después de 3 años de funcionamiento en el Laboratorio Central de Ciments Français, el espectrómetro PW 1210 se ha conectado a un calculador PR 8000, lo que ha permitido doblar fácilmente su rendimiento de utilización. El personal ocupado en el conjunto del análisis por el método de las perlas, incluidos cálculos y dactilografía, se ha reducido en un tercio.

La precisión de los resultados parece superior a la de los métodos químicos corrientes.

En estas condiciones se puede fácilmente demostrar que el análisis automático con ayuda de un espectrómetro de rayos $\mathrm{X}$ conectado a un calculador electrónico da a los laboratorios posibilidades aumentadas de intervención en los reglajes y controles de fabricación, por el número, rapidez de ejecución y precisión de las determinaciones.

\section{bibliografía}

HANS, A.: Coloquio de Espectrometria de rayos $X$. Grenoble, 1959. "Analyse rapide des produits metallurgiques par fluorescence des Rayons X".

Alegre, R.: Coloquio de análisis espectroquimico por rayos $X$. Milán, 1961. "Utilisations de la spectrométrie $\mathrm{X}$ dans l'Industrie cimentière".

MerX, H. y Von Euw, M.: Jornadas del C.E.R.I.L.H., 1964. "Application de la fluorescence X à l'analyse des ciments-Utilisation d'etalons synthétiques". 